\title{
Adaptive Space Mapping with Convergence Enhancement for Optimization of Microwave Structures and Devices
}

\author{
Slawomir Koziel, Senior Member, IEEE, John W. Bandler, Life Fellow, IEEE, \\ and Qingsha S. Cheng, Member, IEEE
}

\begin{abstract}
School of Science and Engineering, Reykjavik University, Kringlunni 1, IS-103 Reykjavik, Iceland Department of Electrical and Computer Eng., McMaster University, Hamilton, ON, Canada L8S 4K1
\end{abstract}

\begin{abstract}
A novel space mapping algorithm is presented that adaptively adjusts the type of space mapping surrogate model used in a given iteration, based on the approximation and generalization capabilities of the model, its ability to satisfy the design specifications, as well as convergence properties of the iterative optimization process. The new technique allows us to avoid a wrong choice of space mapping surrogate which might lead to poor performance of the space mapping algorithm. No extra fine model evaluations are necessary as the assessment process uses only data emerging naturally during the optimization procedure. The performance of the method is verified using microwave design optimization examples and is compared with the previously published adaptive space mapping algorithm.
\end{abstract}

Index Terms - Computer-aided design (CAD), EM optimization, space mapping, adaptive surrogate model, microwave design.

\section{INTRODUCTION}

Space mapping (SM) addresses the problem of optimization of expensive functions, also called "fine" models (typically implemented with CPU-intensive EM simulators), through iterative optimization and updating of the surrogate models which are built using cheaper "coarse" models (e.g., equivalent circuits of the corresponding device) [1], [2]. SM has proved to be successful in many areas (e.g., [1]-[5]).

A common problem in SM optimization is a good choice of the mapping type. Available mappings include input, implicit and different variations of output SM, as well as a frequency SM [2]. They can be combined to adjust the flexibility of the SM surrogate model, which is correlated with the number and type of SM parameters. The surrogate model cannot be too simple; otherwise it will not properly reflect the features of the fine model. Also, it cannot be over-flexible because its generalization properties would be too poor to allow accurate prediction of the fine model

This work was supported in part by the Natural Sciences and Engineering Research Council of Canada under Grants RGPIN7239-06 and STPGP336760-06, and by Bandler Corporation.

S. Koziel was with the Simulation Optimization Systems Research Laboratory, Department of Electrical and Computer Engineering, McMaster University, Hamilton, ON, Canada L8S 4K1. He is now with the School of Science and Engineering, Reykjavik University, Kringlunni 1, IS-103 Reykjavik, Iceland.

Q.S. Cheng is with the Simulation Optimization Systems Research Laboratory, Department of Electrical and Computer Engineering, McMaster University, Hamilton, ON, Canada L8S 4K1.

J.W. Bandler is with the Simulation Optimization Systems Research Laboratory, Department of Electrical and Computer Engineering, McMaster University, Hamilton, ON, Canada L8S 4K1 and also with Bandler Corporation, Dundas, ON, Canada L9H 5E7. response in the neighborhood of the current iteration point. Unfortunately, it is difficult to tell beforehand which combination of mappings may be best for a given problem. A bad choice of mapping may lead to poor performance of the SM algorithm.

In [6], an adaptive technique is presented that allows automatic choice of the surrogate model based on approximation and generalization capabilities estimated using available fine model data. The method [6] overcomes, to some extent, the problem of the proper choice of the surrogate model but it does not take into account other factors such as ability of the surrogate model to satisfy the design specifications and, even more crucial [7], convergence properties of the SM algorithm for a given model.

Here we present an enhancement of our algorithm [6]. This algorithm automatically adjusts the surrogate model type used in a given iteration based not only on the approximation/ generalization capability of the model (as in [6]) but also on the factors mentioned in the previous paragraph, i.e., the estimated convergence properties of the iterative optimization process, and the flexibility of the surrogate model in terms of being capable of satisfying the design specifications.

\section{ADAPTIVE SpACE MAPPING OPTIMIZATION ALGORITHM WITH CONVERGENCE ENHANCEMENT}

Let $\boldsymbol{R}_{f}$ denote the response vector of a fine model of the device of interest. Our goal is to solve

$$
\boldsymbol{x}_{f}^{*}=\arg \min _{\boldsymbol{x}} U\left(\boldsymbol{R}_{f}(\boldsymbol{x})\right)
$$

where $U$ is a given objective function. We consider an optimization algorithm that generates a sequence of points $\boldsymbol{x}^{(i)}$, $i=0,1,2, \ldots$, and a family of surrogate models $\boldsymbol{R}_{s}^{(i)}$, so that

$$
\boldsymbol{x}^{(i+1)}=\arg \min _{\boldsymbol{x},\left\|\boldsymbol{x}-\boldsymbol{x}^{(i)}\right\| \leq \delta^{(i)}} U\left(\boldsymbol{R}_{\mathrm{s}}^{(i)}(\boldsymbol{x})\right)
$$

with $\delta^{(i)}$ being the trust region (TR) radius at iteration $i$. We use a TR method [8] to ensure convergence of the algorithm.

Let $\boldsymbol{R}_{c}$ denote the response vector of the coarse model that describes the same device as the fine model: less accurate but much faster to evaluate. Surrogate models are constructed from the coarse model so that the misalignment between $\boldsymbol{R}_{s}^{(i)}$ and the fine model is minimized. $\boldsymbol{R}_{s}^{(i)}$ is defined as

$$
\boldsymbol{R}_{s}^{(i)}(\boldsymbol{x})=\overline{\boldsymbol{R}}_{s}\left(\boldsymbol{x}, \boldsymbol{p}^{(i)}\right)
$$

where $\overline{\boldsymbol{R}}_{s}$ is a generic SM surrogate model, i.e., the coarse model composed with suitable SM transformations, and

$$
\boldsymbol{p}^{(i)}=\arg \min _{\boldsymbol{p}} \sum_{k=0}^{i}\left\|\boldsymbol{R}_{f}\left(\boldsymbol{x}^{(k)}\right)-\overline{\boldsymbol{R}}_{s}\left(\boldsymbol{x}^{(k)}, \boldsymbol{p}\right)\right\|
$$


is a vector of model parameters. Examples of surrogate models are shown in Table I (Section III.B).

The general SM algorithm (2)-(4) allows us to use various surrogate models. However, a good choice of mapping is usually problem dependent and difficult to make beforehand. Here, we describe a technique for an automatic choice of the suitable surrogate model. The method is adaptive in the sense that it can change the space mapping type used from iteration to iteration based on the following criteria: (i) estimated approximation and generalization capability of the surrogate model, (ii) convergence properties of the optimization process, and (iii) ability of the surrogate model to satisfy the design specifications. The algorithm proposed in [6] used only the first of these criteria: it will be used in this paper as a reference method for comparison purposes.

Let $R_{S}=\left\{\boldsymbol{R}_{s .1} \ldots \boldsymbol{R}_{s . K}\right\}$ be a set of candidate surrogate models. We shall denote by $\boldsymbol{p}_{j}^{0}$ the set of initial values of the parameters of candidate model $\boldsymbol{R}_{s . j}, j=1, \ldots, K$. The model $\boldsymbol{R}_{s, j}^{(i)}(\cdot)=\boldsymbol{R}_{s, j}\left(\cdot, \boldsymbol{p}_{j}^{(i)}\right)$ is set up by proper choice of its parameter values $\boldsymbol{p}_{j}^{(i)}$, which are determined using the parameter extraction procedure

$$
\boldsymbol{p}_{j}^{(i)}=\arg \min _{\boldsymbol{p} \in X_{p . j}} \sum_{\boldsymbol{y} \in X_{A p P}^{(i)}}\left\|\boldsymbol{R}_{f}(\boldsymbol{y})-\boldsymbol{R}_{s . j}(\boldsymbol{y}, \boldsymbol{p})\right\|
$$

where $X_{A P P}^{(i)}$ is a subset of $X^{(i)}=\left\{\boldsymbol{x}^{(0)}, \boldsymbol{x}^{(1)}, \ldots, \boldsymbol{x}^{(i)}\right\}$, the set of all previous iteration points. Let $X_{G E N}^{(i)} \subset X^{(i)}$, such that $X_{A P P}^{(i)} \cap X_{G E N}^{(i)}=\varnothing$. Now, let us define two coefficients:

$F_{A P P . j}^{(i)}=\sum_{y \in X_{A p P}^{(i)}}\left\|\boldsymbol{R}_{f}(\boldsymbol{y})-\boldsymbol{R}_{s . j}\left(\boldsymbol{y}, \boldsymbol{p}_{j}^{0}\right)\right\|^{2} / \sum_{\boldsymbol{y} \in X_{A p P}^{(i)}}\left\|\boldsymbol{R}_{f}(\boldsymbol{y})-\boldsymbol{R}_{s . j}^{(i)}(\boldsymbol{y})\right\|^{2}$

$$
\underset{G \in N . j}{\stackrel{\text { and }}{(i)}}=\sum_{y \in X_{G \in N}^{(i)}}\left\|\boldsymbol{R}_{f}(\boldsymbol{y})-\boldsymbol{R}_{s . j}\left(\boldsymbol{y}, \boldsymbol{p}_{j}^{0}\right)\right\|^{2} / \sum_{\boldsymbol{y} \in X_{G \in \mathbb{N}}^{(i)}}\left\|\boldsymbol{R}_{f}(\boldsymbol{y})-\boldsymbol{R}_{s . j}^{(i)}(\boldsymbol{y})\right\|^{2}
$$

If $X_{G E N}^{(i)}$ is empty, we set $F_{G E N . j}^{(i)}=F_{A P P . j}^{(i)}$.

The first factor, $F_{A P P . j}^{(i)}$, measures the quality of the approximation properties of model $\boldsymbol{R}_{s . j}$, because it is the ratio of the matching error before and after parameter extraction, calculated for the points which were used in parameter extraction. The second factor, $F_{G E N . j}^{(i)}$, measures the quality of the generalization properties of model $\boldsymbol{R}_{s . j}$, because it is the ratio of the matching error before and after parameter extraction, calculated for the points which were not used in extraction. Let $F_{j}^{(i)}$ be a combined quality factor defined as

$$
F_{j}^{(i)}=\alpha F_{A P P . j}^{(i)}+(1-\alpha) F_{G E N . j}^{(i)}
$$

A good surrogate model exhibits high values for both $F_{A P P . j}^{(i)}$ and $F_{G E N . j}^{(i)}$, however, we consider generalization properties as more important and, therefore, we use small values of $\alpha$ (e.g., $\alpha=0.1$ ) [6]. In [6], the choice of the surrogate model $\boldsymbol{R}_{s}^{(i)}$ at any given iteration has been made based on the value of $F_{j}^{(i)}$, in particular we had $\boldsymbol{R}_{s}^{(i)}=\boldsymbol{R}_{s . j_{\max }^{(i)}}^{(i)}$ where $j_{\max }=\arg \max _{j \in\{1,2, \ldots, K\}} F_{j}^{(i)}$.

Here, we enhance this adaptive scheme by considering estimated convergence properties of the iterative optimization process, and the ability of the surrogate model to satisfy the design specifications. In particular, each of the candidate models $\boldsymbol{R}_{s . j}^{(i)}(\cdot)=\boldsymbol{R}_{s . j}\left(\cdot, \boldsymbol{p}_{j}^{(i)}\right)$ is optimized

$$
\boldsymbol{x}_{s, j}^{(i)}=\arg \min _{\boldsymbol{x},\left\|x-\boldsymbol{x}^{(i)}\right\| \leq \delta^{(i)}} U\left(\boldsymbol{R}_{s . j}^{(i)}(\boldsymbol{x})\right)
$$

and the following factors are calculated

$$
\begin{gathered}
D_{j}^{(i)}=\frac{\left\|\boldsymbol{x}^{(i)}-\boldsymbol{x}^{(i-1)}\right\|}{\left\|\boldsymbol{x}_{s . j}^{(i)}-\boldsymbol{x}^{(i)}\right\|} \text { if } i>1 \text { or } \frac{\left\|\boldsymbol{x}^{(1)}\right\|}{\left\|\boldsymbol{x}_{s, j}^{(1)}-\boldsymbol{x}^{(1)}\right\|} \text { otherwise } \\
U_{j}^{(i)}=U\left(\boldsymbol{R}_{s . j}^{(i)}\left(\boldsymbol{x}_{s . j}^{(i)}\right)\right)
\end{gathered}
$$

Factor $D_{j}^{(i)}$ measures the convergence property of the algorithm when using model $\boldsymbol{R}_{s, j}^{(i)}$. The higher the value of $D_{j}^{(i)}$, the larger the ratio between $\left\|\boldsymbol{x}^{(i)}-\boldsymbol{x}^{(i-1)}\right\|$ and $\left\|\boldsymbol{x}_{s . j}{ }^{(i)}-\boldsymbol{x}^{(i)}\right\|$, i.e., the algorithm using the model $\boldsymbol{R}_{s, j}^{(i)}$ is likely to converge faster. $U_{j}^{(i)}$ is the value of the specification error for the optimized model $\boldsymbol{R}_{s . j}^{(i)}$, which may be used to estimate the specification error for the fine model at $\boldsymbol{x}_{s . j}{ }^{(i)}$, provided that $\boldsymbol{R}_{s . j}^{(i)}$ exhibits sufficient matching capability (measured by $F_{j}^{(i)}$ ).

Having this in mind, for each candidate surrogate mode $\boldsymbol{R}_{s . j}{ }^{(i)}$ we define a figure of merit $H_{j}^{(i)}$ as follows

$$
H_{j}^{(i)}=F_{j}^{(i)} \cdot\left(D_{j}^{(i)}\right)^{\beta} \cdot\left(\exp \left(-U_{j}^{(i)}\right)\right)^{\gamma}
$$

In (12), we use $\exp \left(-U_{j}^{(i)}\right)$ instead of $U_{j}^{(i)}$ because we want to transform all possible values of the specification error into positive numbers and have the larger values of factor $H_{j}^{(i)}$ corresponding to smaller values of $U_{j}^{(i)}$ as we are dealing with a minization problem (cf. (1)). Having factors $H_{j}^{(i)}$ we choose the most suitable surrogate model at iteration $i$ as follows: $\boldsymbol{R}_{s}^{(i)}=\boldsymbol{R}_{s . j_{\max }^{(i)}}$, where $j_{\max }=\arg \max _{j \in\{1,2, \ldots, K\}} H_{j}^{(i)}$. In fact, because all candidate surrogate models have been optimized in order to calculate $D_{j}^{(i)}$ and $U_{j}^{(i)}$, we can simply set

$$
\boldsymbol{x}^{(i+1)}=\boldsymbol{x}_{\mathrm{s} . j_{\max }^{(i)}}
$$

with $\boldsymbol{x}_{s, j}{ }^{(i)}$ given by (9) and $j_{\max }$ defined as above.

Parameters $\beta$ and $\gamma$ determine to what extent the factors $D_{j}^{(i)}$ and $U_{j}^{(i)}$ influence the choice of the surrogate model. For $\beta=\gamma=0$ we are back to the algorithm [6]. The adaptive SM algorithm (5)-(13) can be summarized as follows.

Step 0 Set $i=0$; Choose the candidate model set $R_{S}$;

Step 1 Given $X^{(i)}=\left\{\boldsymbol{x}^{(0)}, \boldsymbol{x}^{(1)}, \ldots, \boldsymbol{x}^{(i)}\right\}$, set $X_{A P P}^{(i)}$ and $X_{G E N}^{(i)}$;

Step 2 Perform parameter extraction, obtain models $\boldsymbol{R}_{s . j}{ }^{(i)}$, and calculate quality factors $F_{j}^{(i)}$ as in (8);

Step 3 Optimize models $\boldsymbol{R}_{s . j}{ }^{(i)}$ and obtain $\boldsymbol{x}_{s . j}{ }^{(i)}, D_{j}{ }^{(i)}$, and $U_{j}^{(i)}$ using (9), (10), and (11), respectively;

Step 4 Calculate figures of merit $H_{j}^{(i)}$ as in (12);

Step 5 Set $\boldsymbol{x}^{(i+1)}$ as in (13);

Step 6 Evaluate $\boldsymbol{R}_{f}$ at $\boldsymbol{x}^{(i+1)}$;

Step 7 Update $\delta^{(i)}$;

Step 8 If $\boldsymbol{x}^{(i+1)}$ is accepted set $i=i+1, \delta^{(i)}=\delta^{(i-1)}$ and go to Step 1; else go to Step 3;

Step 9 If the termination condition is not satisfied go to Step 1; else terminate the algorithm.

Our algorithm does not require any extra fine model evaluations because the surrogate model assessment is based on 
already existing fine model data. Additional computational effort concerns the coarse model only, and does not substantially affect the total execution time of the optimization algorithm as the coarse model is assumed to be cheap. On the other hand, because the surrogate model assessment is based on additional criteria including convergence properties and model flexibility, it is expected that our algorithm will exhibit performance comparable or better than the algorithm [6].

\section{VERIFICATION EXAMPLES}

\section{A. Test Problem Description}

Problem 1: Bandstop microstrip filter with open stubs [9] (Fig. 1). The fine model is simulated with Sonnet's em [10] using a grid with a $0.2 \mathrm{mil} \times 1 \mathrm{mil}$ cell size. The coarse model, Fig. 2, is the equivalent circuit implemented in Agilent ADS [11]. The design parameters are $\boldsymbol{x}=\left[W_{1} W_{2} L_{0} L_{1} L_{2}\right]^{T}$. The design specs are $\left|S_{21}\right| \leq 0.05$ for $9.4 \mathrm{GHz} \leq \omega \leq 10.6 \mathrm{GHz}$, and $\left|S_{21}\right| \geq 0.9$ for $5 \mathrm{GHz} \leq \omega \leq 8 \mathrm{GHz}$ and $12 \mathrm{GHz} \leq \omega \leq 15 \mathrm{GHz}$.

Problem 2: Seven-section capacitively-loaded impedance transformer [12]. Both "coarse" and "fine" models (Fig. 3) are implemented in Matlab. The design parameters are

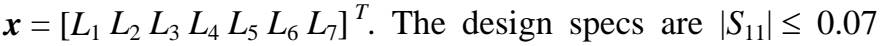
for $1.0 \mathrm{GHz} \leq \omega \leq 7.7 \mathrm{GHz}$.

Problem 3: Second-order capacitively-coupled dualbehavior resonator (CCDBR) microstrip filter [13] (Fig. 4). The design parameters are $\boldsymbol{x}=\left[L_{1} L_{2} L_{3} S\right]^{T}$. The fine model is simulated in FEKO [14]. The coarse model, Fig. 5, is implemented in Agilent ADS [11]. The design specifications are $\left|S_{21}\right| \geq-3 \mathrm{~dB}$ for $3.8 \mathrm{GHz} \leq \omega \leq 4.2 \mathrm{GHz}$, and $\left|S_{21}\right| \leq-20 \mathrm{~dB}$ for $2.0 \mathrm{GHz} \leq \omega \leq 3.2 \mathrm{GHz}$ and $4.8 \mathrm{GHz} \leq \omega \leq 6.0 \mathrm{GHz}$.

\section{B. Experimental Setup}

For Problems 1-3 we performed SM optimization using the adaptive SM algorithm introduced in Section II. We use $\alpha=0.1$ and two settings for $\beta$ and $\gamma$ : (i) $\beta=\gamma=0$, which corresponds to the algorithm [6] used here as a reference method, and (ii) $\beta=\gamma=1$, which corresponds to the algorithm of Section II with factors $D_{j}^{(i)}$ and $U_{j}^{(i)}$ taken into account in a nontrivial way. For all problems we use the same candidate model set $\{\boldsymbol{c d}, \boldsymbol{d F}, \boldsymbol{c d F}$, $\boldsymbol{B} \boldsymbol{c d}\}$. The model naming convention is explained in Table I. The test set $X_{A P P}^{(i)}\left(X_{G E N}^{(i)}\right)$ is chosen to contain about 2/3 (1/3) of available points (cf. [6]).

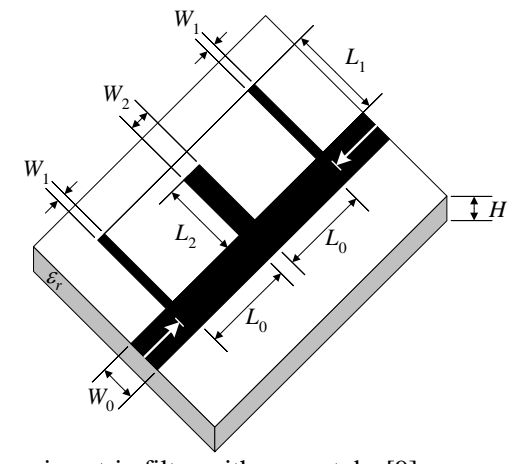

Fig. 1. Bandstop microstrip filter with open stubs [9].

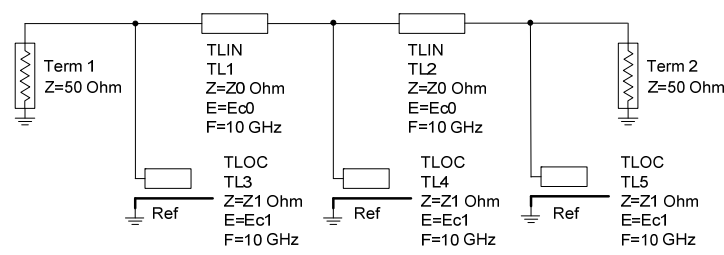

Fig. 2. Coarse model of the bandstop microstrip filter (Agilent ADS).

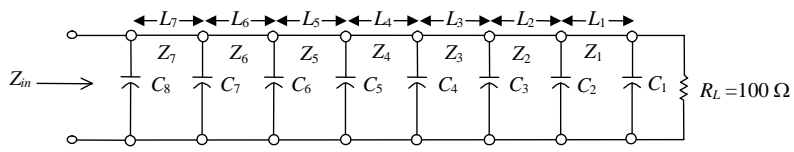

(a)

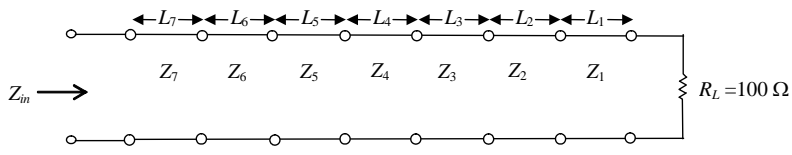

(b)

Fig. 3. Seven-section capacitively-loaded impedance transformer: "fine" model (a) and "coarse" model (b) [12].

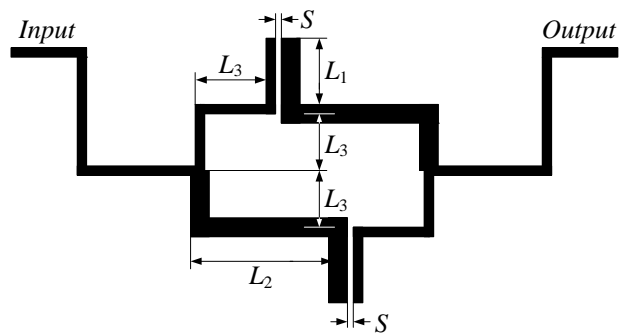

Fig. 4. Geometry of the CCDBR microstrip filter [13].

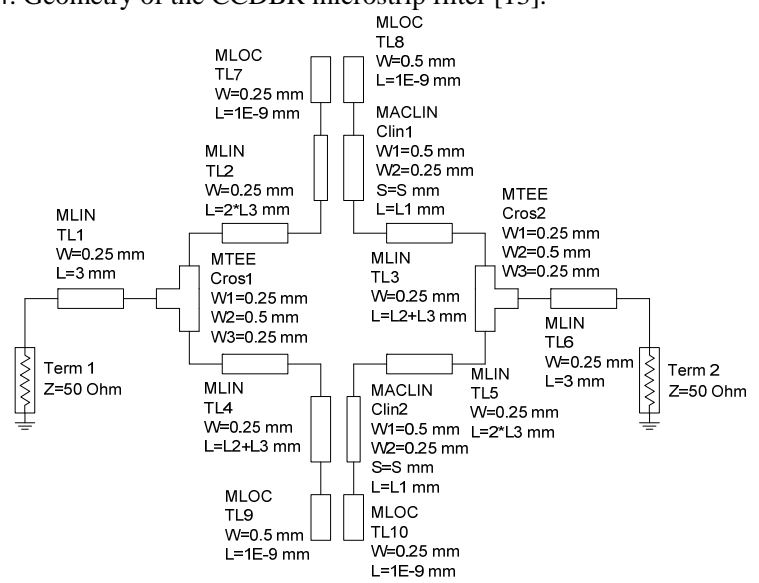

Fig. 5. Coarse model of the CCDBR microstrip filter (Agilent ADS).

TABLE I

NAMING CONVENTION FOR SM SURROGATE MODELS ${ }^{1}$

\begin{tabular}{|c|c|c|}
\hline Name & Definition & Description \\
\hline$C$ & $\boldsymbol{R}_{c}(\boldsymbol{x}+\boldsymbol{c})$ & Input SM with $c$ being $n \times 1$ vector $^{2}$ \\
\hline B & $\boldsymbol{R}_{c}(\boldsymbol{B} \cdot \boldsymbol{x})$ & Input SM with $\boldsymbol{B}$ being $n \times n$ matrix ${ }^{2}$ \\
\hline$F$ & $\boldsymbol{R}_{c}(\boldsymbol{x}, \boldsymbol{F})$ & $\begin{array}{l}\text { Frequency SM with coarse model evaluated at } \\
\text { frequencies different from the original sweep } \\
\text { according to } \omega \rightarrow f_{1}+f_{2} \omega \text {, with } \boldsymbol{F}=\left[f_{1} f_{2}\right]^{T}\end{array}$ \\
\hline$d$ & $\boldsymbol{R}_{c}(\boldsymbol{x})+\boldsymbol{d}$ & $\begin{array}{l}\text { Output SM with } \boldsymbol{d} \text { being } m \times 1 \text { vector }^{3} \text {; at } \\
\text { iteration } i, \boldsymbol{d} \text { is calculated as } \boldsymbol{d}=\boldsymbol{R}_{f}\left(\boldsymbol{x}^{(i)}\right)-\boldsymbol{R}_{c}\left(\boldsymbol{x}^{(i)}\right)\end{array}$ \\
\hline
\end{tabular}

${ }^{1}$ Combined models are obtained as combinations of the corresponding definitions, e.g., $\boldsymbol{c d}$ is defined as $\boldsymbol{R}_{c}(\boldsymbol{x}+\boldsymbol{c})+\boldsymbol{d}, \boldsymbol{B} \boldsymbol{c}$ is defined as $\boldsymbol{R}_{c}(\boldsymbol{B} \cdot \boldsymbol{x}+\boldsymbol{c})$, etc.

${ }^{2} n$ is the number of the design variables

${ }^{3} \mathrm{~m}$ is the dimension of the model response 


\section{Experimental Results and Discussion}

Table II shows the results of our experiments, i.e., the objective function value (specification error) and the number of fine model evaluations necessary to obtain the solution for problems 1-3. Figs. 6-8 show the initial fine model response and the response at the final solution found by the algorithm (5)-(13). It is seen that in Problem 1 both algorithms exhibit the same performance, which indicates that the same surrogate models are chosen in subsequent iterations of the algorithm in both cases. For Problem 2, the new algorithm yields slightly better results than the algorithm [6] with a smaller number of fine model evaluations. For Problem 3, the new algorithm yields a substantially better solution than the algorithm [6] with a similar number of fine model evaluations. Thus, the overall performance is in favor of the new algorithm.

\section{CONCLUSION}

An adaptive SM algorithm with convergence enhancement is presented. At any iteration, the algorithm automatically chooses the most suitable surrogate model based on several criteria including approximation/generalization capability of the model as well as the estimated convergence properties of the optimization process. As verified with several microwave design optimization problems, the performance of the SM optimization process is improved with respect to the previously published adaptive SM technique.

\section{REFERENCES}

[1] J.W. Bandler, Q.S. Cheng, S.A. Dakroury, A.S. Mohamed, M.H. Bakr, K. Madsen, and J. Søndergaard, "Space mapping: the state of the art," IEEE Trans. Microwave Theory Tech., vol. 52, no. 1, pp. 337-361, Jan. 2004.

[2] S. Koziel, J.W. Bandler, and K. Madsen, "A space mapping framework for engineering optimization: theory and implementation," IEEE Trans. Microwave Theory Tech., vol. 54, no. 10, pp. 3721-3730, Oct. 2006.

[3] H.-S. Choi, D. H. Kim, I. H. Park, and S. Y. Hahn, "A new design technique of magnetic systems using space mapping algorithm," IEEE Trans. Magn., vol. 37, no. 5, pp. 3627-3630, Sept. 2001.

[4] M. Redhe and L. Nilsson, "Optimization of the new Saab 9-3 exposed to impact load using a space mapping technique," Structural and Multidisciplinary Optimization, vol. 27, pp. 411-420, 2004.

[5] G. Crevecoeur, L. Dupre, and R. Van de Walle, "Space mapping optimization of the magnetic circuit of electrical machines including local material degradation," IEEE Trans. Magn., vol. 43, no 6, pp. 26092611, June 2007.

[6] S. Koziel and J.W. Bandler, "Space-mapping optimization with adaptive surrogate model," IEEE Trans. Microwave Theory Tech., vol. 55, no. 3, pp. 541-547, March 2007.

[7] S. Koziel and J.W. Bandler, "Coarse and surrogate model assessment for engineering design optimization with space mapping," IEEE MTT-S Int. Microwave Symp. Dig, Honolulu, HI, 2007, pp. 107-110.

[8] A.R. Conn, N.I.M. Gould, and P.L. Toint, Trust Region Methods, MPSSIAM Series on Optimization, 2000.

[9] M.H. Bakr, J.W. Bandler, M.A. Ismail, J.E. Rayas-Sánchez, and Q.J. Zhang, "Neural space-mapping optimization for EM-based design," IEEE Trans. Microwave Theory Tech., vol. 48, no. 12, pp. 2307-2315, Dec. 2000.

[10] $\boldsymbol{e m}^{\mathrm{TM}}$ Version 10.52, Sonnet Software, Inc., 100 Elwood Davis Road, North Syracuse, NY 13212, USA.

[11] Agilent ADS, Version 2003C, Agilent Technologies, 1400 Fountaingrove Parkway, Santa Rosa, CA 95403-1799, 2003.
[12] M.H. Bakr, J.W. Bandler, K. Madsen, and J. Søndergaard, "An introduction to the space mapping technique," Optimization Eng., vol. 2, no. 4, pp. 369-384, Dec. 2001.

[13] A. Manchec, C. Quendo, J.-F. Favennec, E. Rius, and C. Person, "Synthesis of capacitive-coupled dual-behavior resonator (CCDBR) filters," IEEE Trans. Microwave Theory Tech., vol. 54, no. 6, pp. 23462355, June 2006.

[14] FEKO ${ }^{\circledR}$ User's Manual, Suite 4.2, June 2004, EM Software \& SystemsS.A. (Pty) Ltd, 32 Techno Lane, Technopark, Stellenbosch, 7600, South Africa.

TABLE II

OPTIMIZATION RESULTS FOR PROBLEMS 1-3

\begin{tabular}{cccc}
\hline \hline $\begin{array}{c}\text { Test } \\
\text { Problem }\end{array}$ & SM Algorithm & $\begin{array}{c}\text { Specification } \\
\text { Error }\end{array}$ & $\begin{array}{c}\text { Number of } \\
\text { Fine Model } \\
\text { Evaluations }\end{array}$ \\
\hline \multirow{2}{*}{1} & Adaptive [6] $(\beta=\gamma=0)$ & -0.0192 & 13 \\
& Adaptive (5)-(13) $(\beta=\gamma=1)$ & -0.0192 & 13 \\
\hline \multirow{2}{*}{2} & Adaptive [6] $(\beta=\gamma=0)$ & -0.0083 & 9 \\
& Adaptive (5)-(13) $(\beta=\gamma=1)$ & -0.0085 & 6 \\
\hline \multirow{2}{*}{3} & Adaptive [6] $(\beta=\gamma=0)$ & -0.48 & 12 \\
& Adaptive (5)-(13) $(\beta=\gamma=1)$ & -2.04 & 14 \\
\hline \hline
\end{tabular}

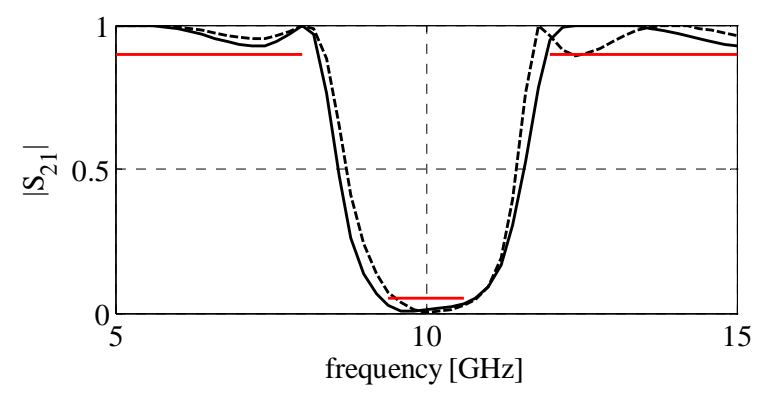

Fig. 6. Problem 1: Initial (dashed line) and optimized (solid line) $\left|S_{21}\right|$ versus frequency for the microstrip band-stop filter.

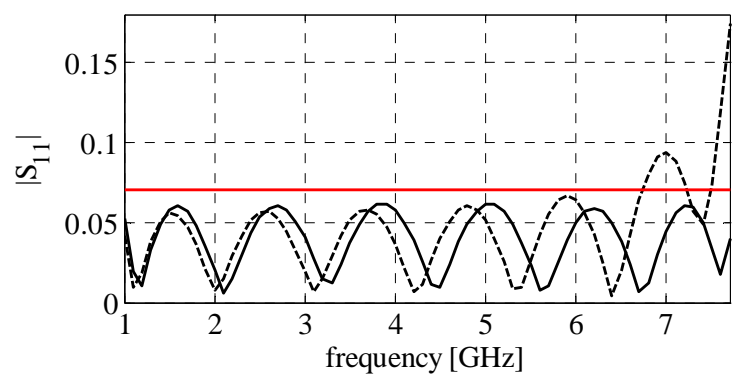

Fig. 7. Problem 2: Initial (dashed line) and optimized (solid line) $\left|S_{11}\right|$ versus frequency for the seven-section impedance transformer.

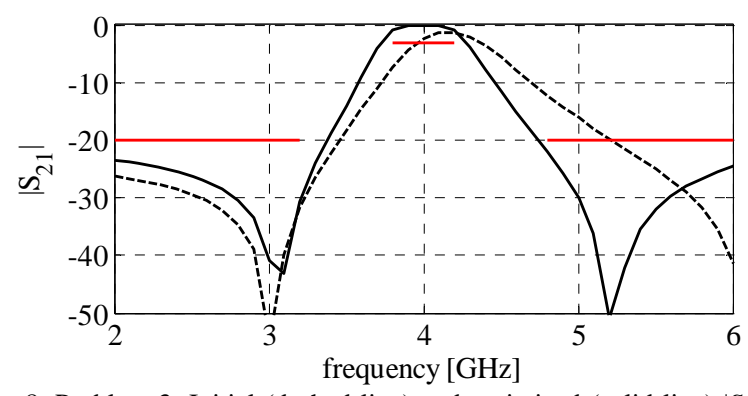

Fig. 8. Problem 3: Initial (dashed line) and optimized (solid line) $\left|S_{21}\right|$ versus frequency for the CCDBR filter. 